\title{
Operations of the LHAASO-WCDA
}

\section{Cheng Liu, ${ }^{a}, *$ Mingjun Chen, ${ }^{a}$ Minghao Gu, ${ }^{a}$ Xiaohao You, ${ }^{a}$ HuiCai Li, ${ }^{a}$ Bo Gao, ${ }^{a}$ Shicong $\mathrm{Hu}^{a}$ and Zhiguo $\mathrm{Yao}^{a}$}

${ }^{a}$ Institute of High Energy Physics, 19B Yuquan Road, Shijingshan District, Beijing, China

E-mail: liuc@ihep.ac.cn

\section{On behalf of LHAASO collaboration}

The Water Cherenkov Detector Array (WCDA) is one of the components of the Large High Altitude Air Shower Observatory (LHAASO). WCDA, divided into 3 separate arrays, will make survey observation on the gamma-ray sky of $100 \mathrm{GeV}-30 \mathrm{TeV}$. The first array $(150 \mathrm{~m} \times$ $150 \mathrm{~m}$ ), denoted as WCDA-1, has already be operated since April 2019 and one more array of the same size, referred to as WCDA-2, has also been in operation since November 2019. The third array, WCDA-3, with a size of $300 \mathrm{~m} \times 110 \mathrm{~m}$, has been tested at beginning of 2021 and the full array of WCDA is being in the testing since March 2021. This paper will describe the operation status of the LHAASO-WCDA since April 2019.

$37^{\text {th }}$ International Cosmic Ray Conference (ICRC 2021)

July 12th-23rd, 2021

Online - Berlin, Germany

\footnotetext{
*Presenter
} 


\section{Introduction}

The Large High Altitude Air Shower Observatory (LHAASO) is a hybrid extensive air shower (EAS) detector arrays sited at Mt. Haizi (29²1' 27.6”N, $100^{\circ} 08^{\prime} 19.6^{\prime}$ E, $4410 \mathrm{~m}$ a.s.1.), in Daocheng, Sichuan province, China. The site of LHAASO is close to the national road G227 and about 10 $\mathrm{km}$ from the Yading airport. The LHAASO consists of three interconnected detector arrays: an EAS array covering an area of $1.3 \mathrm{~km}^{2}$ (KM2A) with 5195 electromagnetic particle detectors (EDs) and 1188 muon detectors (MDs), plus the 78,000 $\mathrm{m}^{2}$ water Cherenkov detector array (WCDA) and an array of 18 wide field-of-view Cherenkov telescopes (WFCTA), as show in Fig. 1 [1]. Civil construction of LHAASO started in the middle of 2016 and the whole array will be completed in July of 2021.

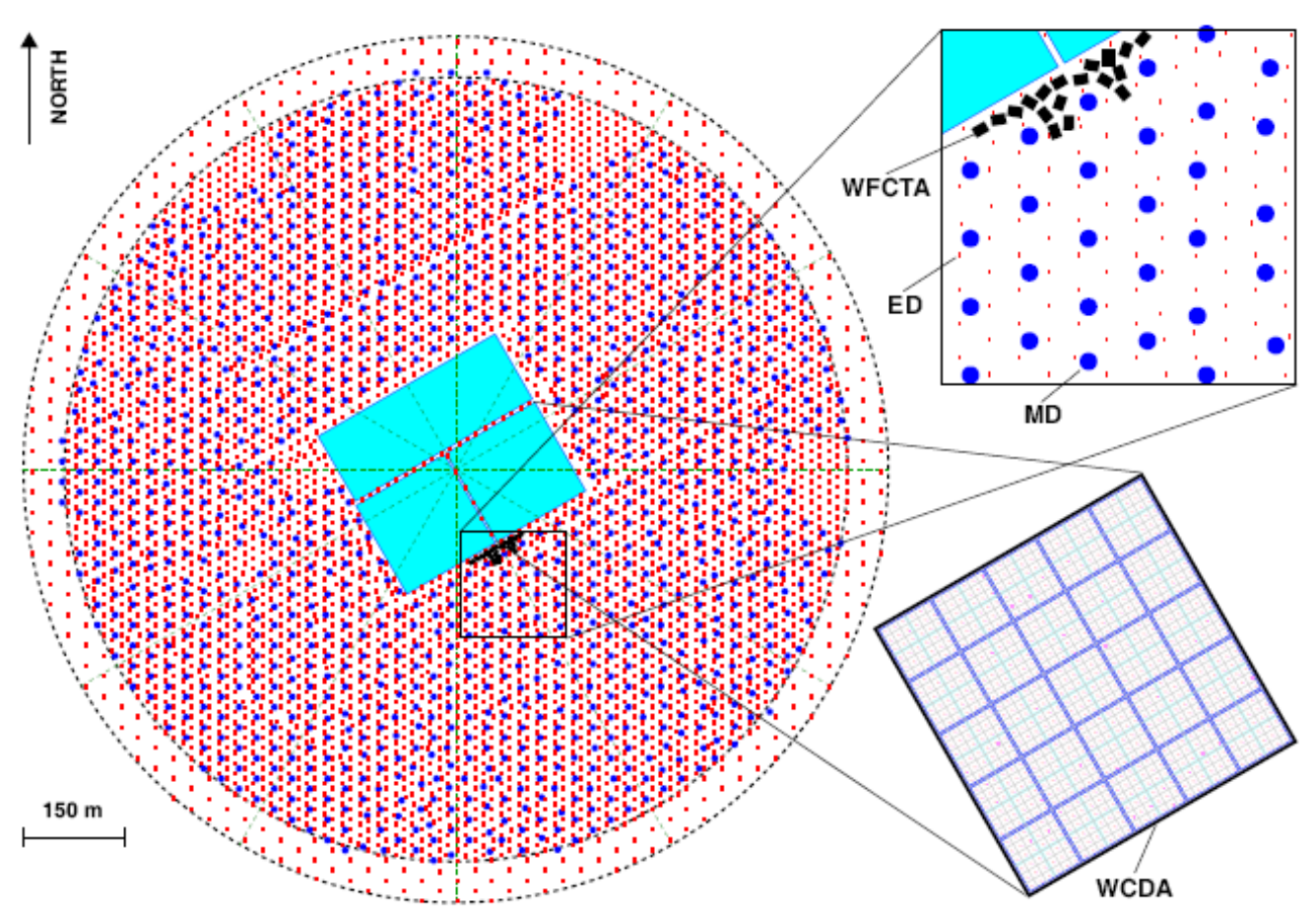

Figure 1: Layout of the LHAASO experiment.

LHAASO is designed for the study of cosmic ray particles with energy ranging from a few tens of $\mathrm{GeV}$ to a few $\mathrm{EeV}$ [2]. The main physics objectives of LHAASO are to explore the origin of high-energy cosmic ray, full sky gamma-ray source survey, and carry out the relevant studies on the basic sciences, such as the high-energy radiation, celestial body evolution, dark matter detection, and so on [3].

The WCDA focuses on surveying the Northern sky for steady and transient sources from $100 \mathrm{GeV}$ to $30 \mathrm{TeV}$, with a very high background rejection power and a good angular resolution. In this paper, the design and configuration of WCDA are described in the second section. The operation status of the WCDA since April 2019 is described in the third section. And a short summary follows at the end of the paper. 


\section{Detector}

At the center of the LHAASO, the WCDA is composed of 3 separate arrays with a water depth of $4.4 \mathrm{~m}$, as show in Fig. 1. Each arrays is divided into $5 \mathrm{~m} \times 5 \mathrm{~m}$ cells partitioned by black plastic curtains to prevent the light passing through the neighboring cells. The first array, denoted as WCDA-1, is a square pond of $150 \mathrm{~m} \times 150 \mathrm{~m}$ consisting of 900 detector cells. It has been put in operation since April 2019 and one more array of the same size, referred to as WCDA-2, has also been in operation since November 2019. The third array, WCDA-3, with a size of $300 \mathrm{~m} \times 110 \mathrm{~m}$, containing 1,320 detector cells, has been tested since the beginning of 2021 .

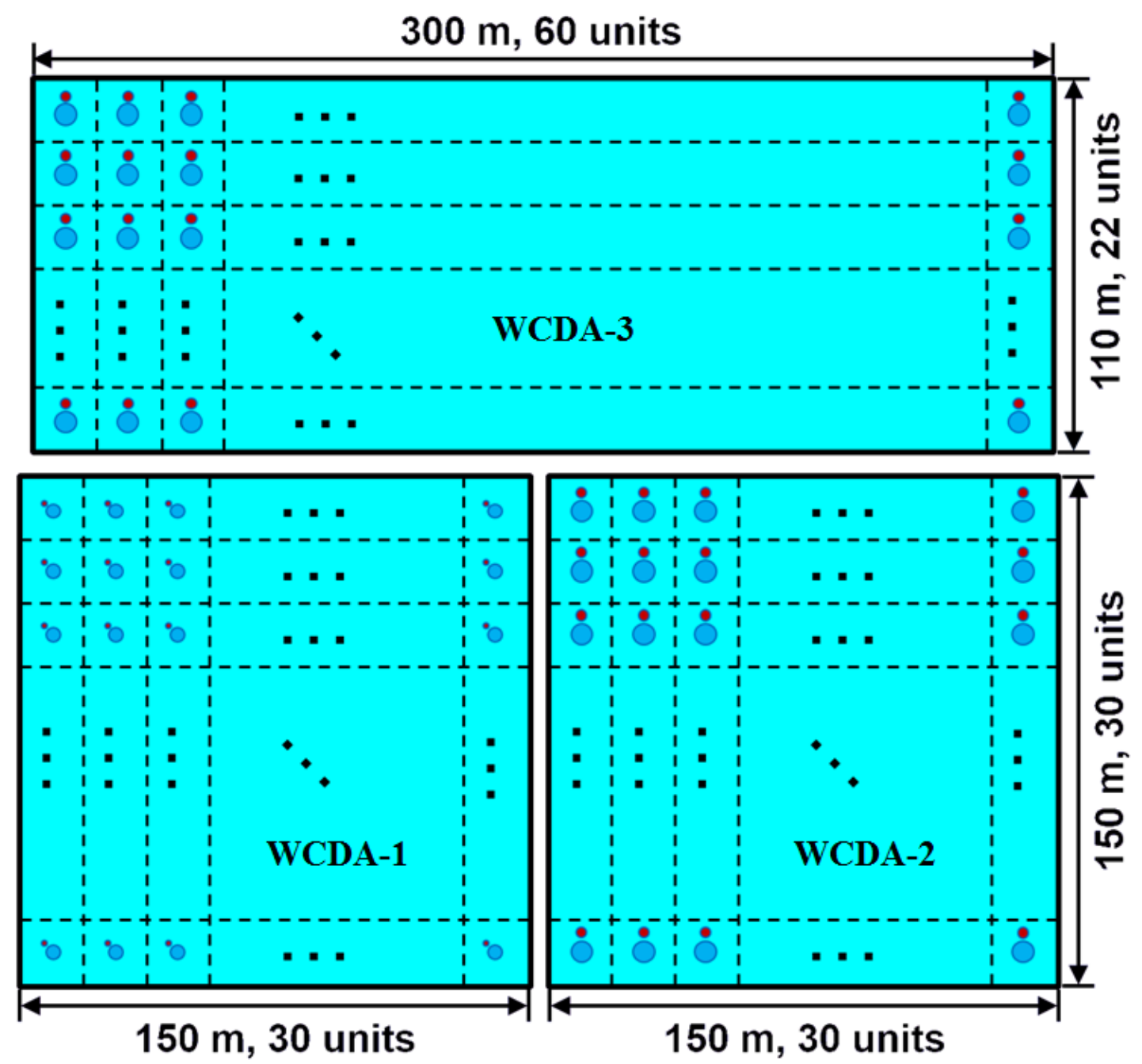

Figure 2: Schematic of the LHAASO-WCDA layout.

In Fig. 2, two dots in each cell indicate two photomultiplier tubes (PMTs). The WCDA-1 is equipped with 8-inch and 1.5-inch PMTs at the bottom center of each cell, and the other two arrays are equipped with 20-inch and 3-inch PMTs. Each PMT is looking upward to collect Cherenkov 
light generated by EAS secondary particles in water. Fig. 3 shows the photograph of the four types of PMTs used in WCDA.
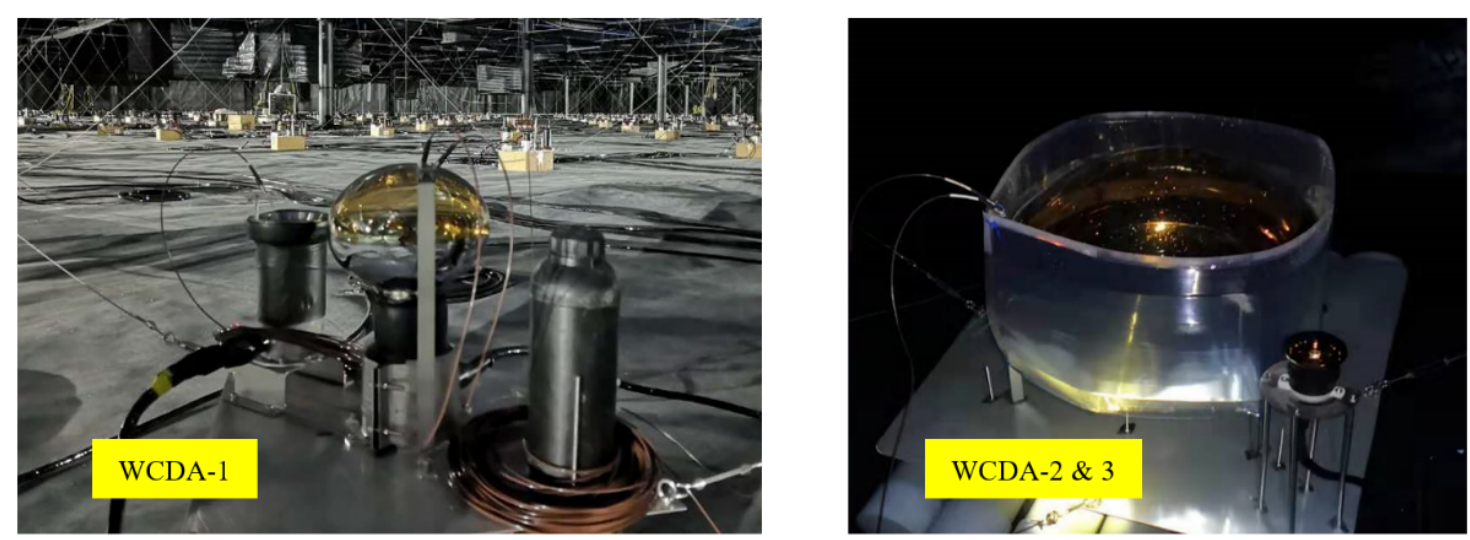

Figure 3: Photograph of the four types of PMTs using in WCDA.

The large PMT (8-inch or 20-inch) in each cell measures the number of photons and arrival time. The 20-inch PMT uses microchannel-plate (MCP) instead of the traditional dynodes and are produced by North Night Vision Tech. Cor. Ltd (NNVT). This enhances the gamma ray detecting sensitivity at energies below $500 \mathrm{GeV}$, thus improved the performance of WCDA as a survey facility for gamma ray bursts (GRBs). Detailed performance of 20-inch PMT will be introduced in this talk [4]. The Transit Time Spread (TTS) of 8-inch PMT is less than 3ns and for 20-inch PMT, the TTS is less than 6.5ns. These large PMTs are calibrated within $0.2 \mathrm{~ns}$ and this enables the shower arrival direction to be measured with a resolution of $0.2^{\circ}$ above a few $\mathrm{TeV}$ [5]. The PMT signals are transmitted through $30 \mathrm{~m}$ long cables to a front-end electronics (FEE) board, which can handle signals from 9 PMTs. To enlarge the dynamic range, small PMTs (1.5-inch or 3-inch) are located beside the large PMTs in each cell. Compared with 8-inch / 20-inch PMT, the receiving area of 1.5-inch / 3-inch PMT will be reduced by 28 / 44 times, which could be used to measure higher energy EAS events. The 1.5-inch PMT is designed to make precise measurements in the core area to identify the composition of cosmic ray events from $100 \mathrm{TeV}$ to $10 \mathrm{PeV}$ [6]. Each cluster in the electronic system receives signals from 36 small PMTs. The main information of PMTs used in WCDA are given in Table 1. The charge calibration method and results of PMTs are presented in this talk [7].

Table 1: PMTs information of LHAASO-WCDA

\begin{tabular}{|l|l|l|l|l|l|}
\hline PMT & TTS (ns) & Dynamic range (PEs) & Manufacturer & Model number & Layout \\
\hline 8-inch & $<3$ & $1-4,000$ & Hamamatsu & CR-365 & WCDA-1 \\
\hline 1.5-inch & - & $20-200,000$ & HZC Photonics & XP-3960 & WCDA-1 \\
\hline 20-inch & $<6.5$ & $1-1,800$ & NNVT & GDB-6203 & WCDA-2\&3 \\
\hline 3-inch & - & $1-3,000$ & HZC Photonics & XP-72B22 & WCDA-2\&3 \\
\hline
\end{tabular}




\section{Operation}

The detector installation of WCDA started in October 2018, and WCDA-1 has been operated since April 2019, while the third array, WCDA-3, has been tested at beginning of 2021. The whole construction and operation process is divided into three phases: (1) 1/4 of WCDA, also called WCDA-1, was completed in April of 2019 and put in operation since then; (2) 1/2 of WCDA, which has been in operation since March 2020 with WCDA-1 and WCDA-2; (3) Full array of WCDA started being in operation in March of 2021. The operation status of these three phases are as follow.

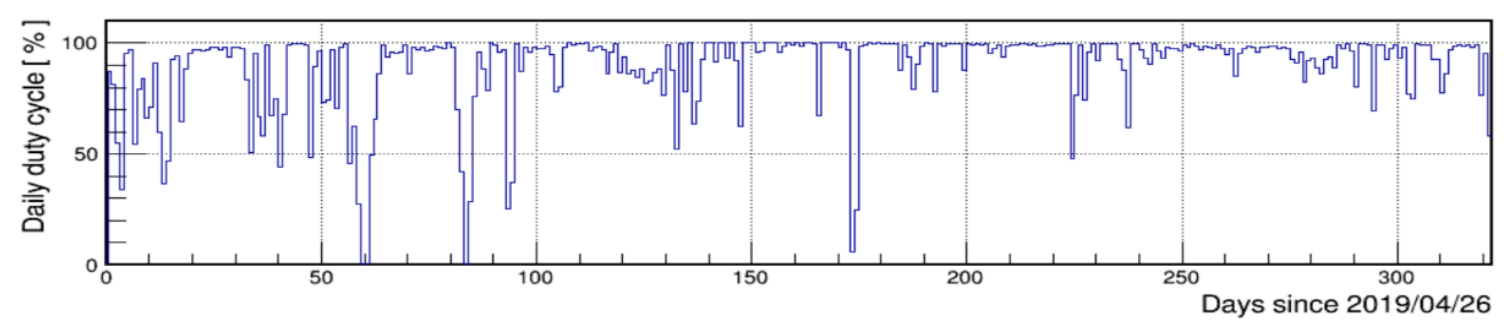

Figure 4: The daily live time of WCDA-1 as a function of days since April 26, 2019.

\subsection{WCDA-1 (2019/4/16 - 2020/3/12)}

At the beginning, a large volume of monitoring data was collected for testing the operation. A trigger algorithm was implemented to record EAS event by requiring at least 20 groups (a group being $3 \times 3$ detector cells) with each group containing at least one simultaneous hit in a time window of $300 \mathrm{~ns}$. In a group, one hit is recorded if there are signals greater than the threshold of $1 / 3 \mathrm{PE}$ in the nine 8-inch PMTs (no matter how many PMTs in total). The EAS event trigger rate is about 20 $\mathrm{kHz}$, while the hit rate of a 8-inch PMT is about $21 \mathrm{kHz}$. For a 1.5-inch PMT, for signals greater than the threshold of $10 \mathrm{PEs}$, the hit rate is about $12 \mathrm{~Hz}$. Once an EAS event trigger is formed, charges recorded by both 8-inch and 1.5-inch PMTs in $2 \mu$ s are read out together with the absolute trigger time to build a complete event. About $3 \mathrm{~TB}$ of raw data are generated every day. In Fig. 4, the daily live time of WCDA-1 are plotted as a function of time since the April 26, 2019. By using the 179 days data, WCDA- 1 is tested by observing the Crab Nebula as the standard candle of the $\mathrm{TeV}$ gamma ray astronomy. The Crab Nebula has been detected with a significance of 77.4 , corresponding to a sensitivity of $65 \mathrm{mCU}$, in agreement with the design specification [2].

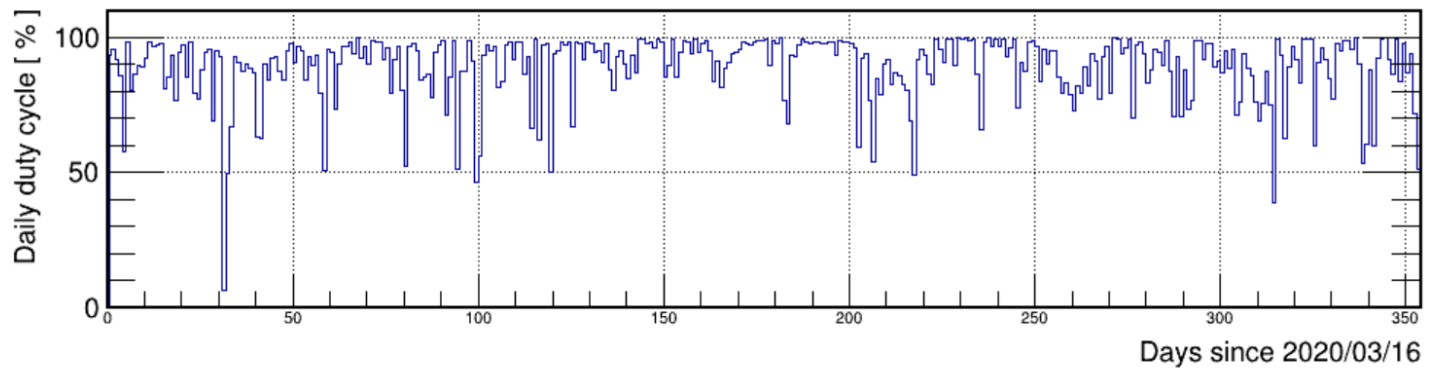

Figure 5: The daily live time of 1/2 WCDA as a function of days since March 16, 2020. 


\subsection{Half array $(2020 / 3 / 16-2021 / 3 / 4)$}

WCDA-2, equipped with 20-inch and 3-inch PMTs, has been in operation independently since November 2019. The photo cathode of 20-inch PMT is a factor of 6.25 larger than the 8-inch PMT, so that the rate has increased significantly. At the beginning, the hit rate of a 20-inch PMT with the threshold of $1 / 3 \mathrm{PE}$ was about $42 \mathrm{kHz}$ without magnetic shielding. After upgrading the data acquisition system (DAQ) and the optimization of trigger algorithm, in March 2020, both WCDA-1 and WCDA-2 were put in science operations.

The 20-inch PMT is greatly affected by the geomagnetic field, the TTS and collection efficiency of PMT become worse without magnetic shielding [4]. Therefor, in October 2020, a magnetic shielding has been added around the 20-inch PMTs in WCDA-2 and the hit rate of the PMT with the threshold of $1 / 3 \mathrm{PE}$ is increased to $55 \mathrm{kHz}$. Taken into account the features of lateral distribution of air showers, in order to reach a low energy threshold, a natural consideration on the trigger algorithm is to use the multiple zoned pattern. Once the number of fired cells in any of these zone exceeds a certain number within a short time window, the whole array is then set to be triggered [8]. For the $1 / 2$ WCDA arrays, the EAS event trigger rate is about $80 \mathrm{kHz}$ and the raw data is about $15 \mathrm{~TB} /$ day. In Fig. 5, the daily live time of 1/2 WCDA arrays are plotted as a function of time since the March 16, 2020.

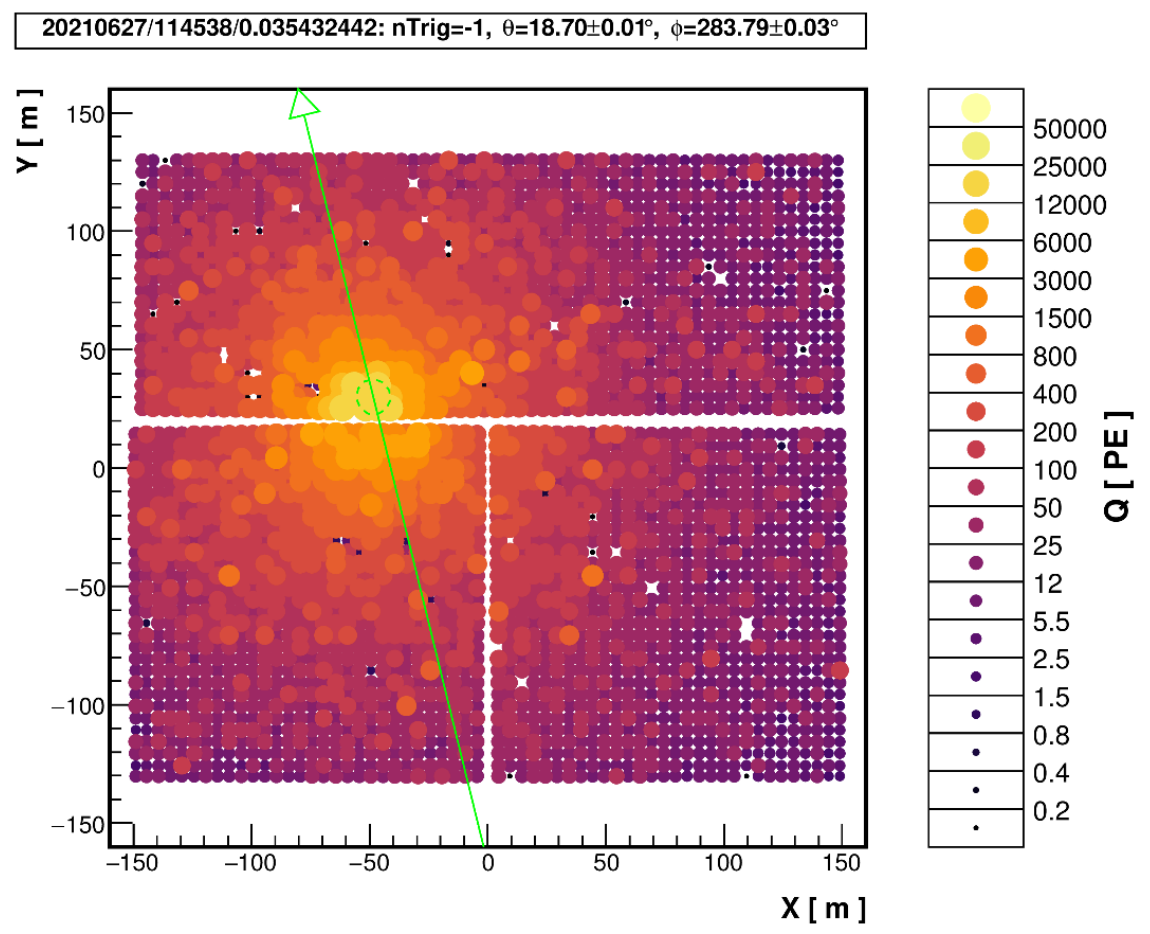

Figure 6: A typical high energy shower event detected by WCDA.

\subsection{Full array (2021/3/5 - now)}

The third array, WCDA-3, has been tested at beginning of 2021 and the full array is being in the testing since March 2021. The full array is split into 72 approximately half-overlapping trigger 
clusters, each containing 144 cells and covering an area of $60 \mathrm{~m} \times 60 \mathrm{~m}$. During commissioning, the multiplicity threshold of the array is set to 30 hits and the 20-inch PMT signal threshold is changed from $1 / 3$ PE to $1 \mathrm{PE}$, in order to reduce the influence of noise. With these conditions, the trigger rate of full array is about $30 \mathrm{kHz}$ and the raw data is about $12 \mathrm{~TB} /$ day. Fig. 6 shows a typical high energy shower event detected by WCDA, where the photoelectrons information of the 4 PMTs is combined.

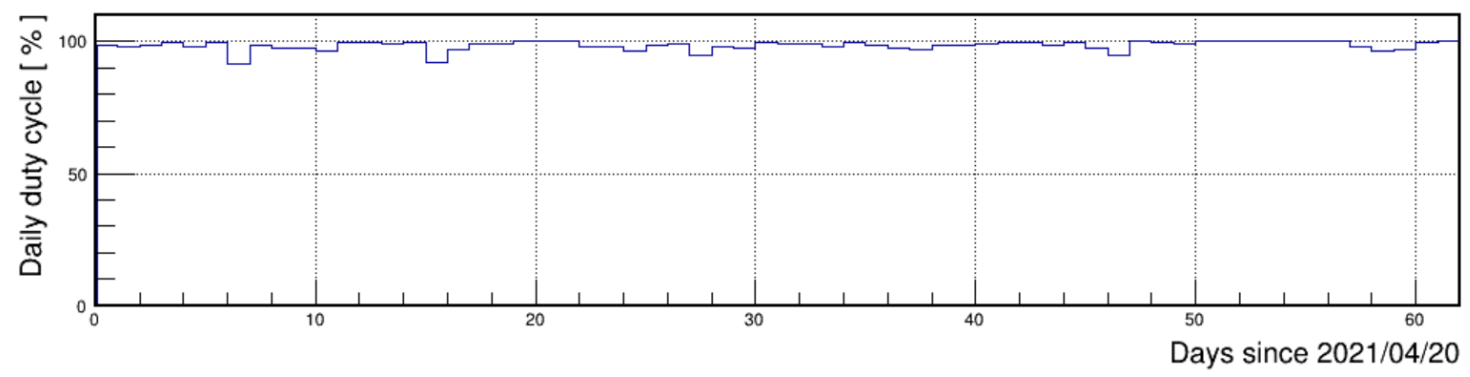

Figure 7: The daily live time of full array as a function of time since the April 20, 2021.

In Fig. 7, the daily live time of WCDA arrays are plotted as a function of time since the April 20, 2021. The operation of the full array has been very stable with a live time more than $98 \%$ and Fig. 8 shows the daily event rate change since then. Due to the change of water quality, the event rate began to decrease from $32 \mathrm{kHz}$ in the first 30 days, and finally stabilized at about $28 \mathrm{kHz}$.

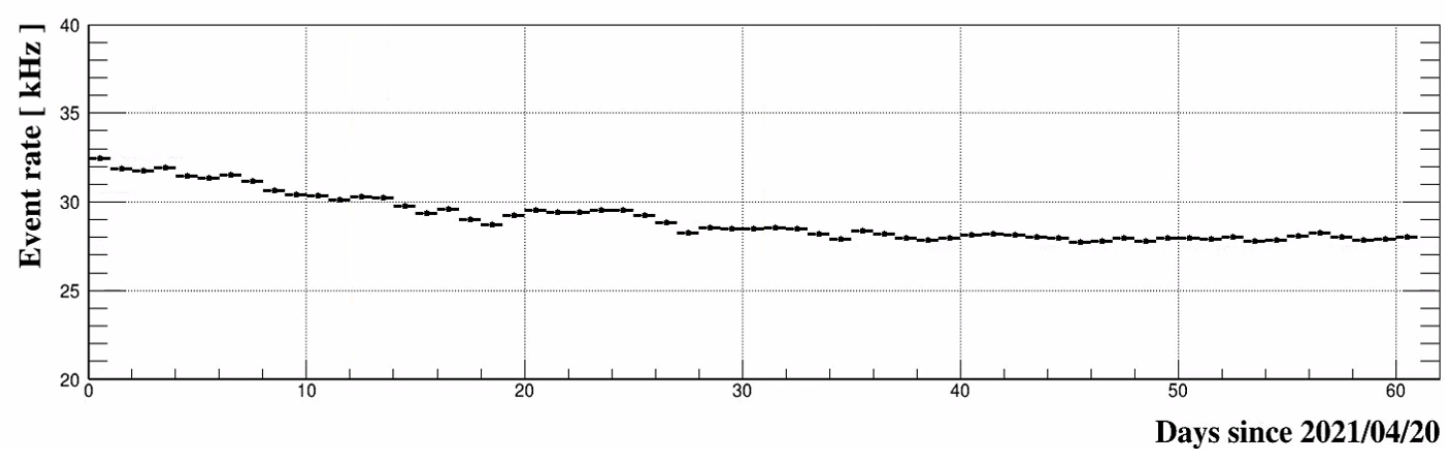

Figure 8: The daily event rate of full array as a function of time since the April 20, 2021.

\section{Summary}

The detector installation of WCDA started from the October 2018, WCDA-1 has already be operated in April, 2019 and the third array, WCDA-3, was test at the beginning of 2021 . The full array of WCDA is being in the testing since March 2021 and runs smoothly. The preliminary analysis results, including observed significant source candidates, the spectrum measurement and other related works are presented in other contribution in this conference [9-13]. 


\section{Acknowledgements}

The authors would like to thank all staff members who work at the LHAASO site above 4400 meters above sea level year-round to maintain the detector and keep the electrical power supply and other components of the experiment operating smoothly. We are grateful to the Chengdu Management Committee of Tianfu New Area for their constant financial support of the science research with LHAASO data..

\section{References}

[1] Huihai He for the LHAASO collaboration. Design of the LHAASO detectors. Radiation Detection Technology and Methods, 2:7, 2018.

[2] The LHAASO collaboration. Performance of LHAASO-WCDA and observation of Crab nebula as a standard candle. e-Print: 2101.03508 [astro-ph.IM].

[3] Zhen Cao et al. Introduction to Large High Altitude Air Shower Observatory. Chinese Astronomy and Astrophysics, 43:457-478, 2019.

[4] Xiaohao You et al. The application of 20 inch PMT in LHASSO-WCDA. 37th ICRC (2021), ID-167.

[5] Bo Gao et al. Time calibration of the LHAASO-WCDA detectors. 37th ICRC (2021), ID-1126.

[6] Cheng Liu et al. The dynamic range extension system for the LHAASO-WCDA experiment. 35th ICRC (2017), CR-1091.

[7] Shicong Hu et al. The charge calibration of LHAASO-WCDA. 37th ICRC (2021), ID-1164.

[8] Xiaojie Wang et al. Trigger and noise filtering algorithms for the LHAASO water Cherenkov detector array. Radiat Detect Technol and Methods, 1:14, 2017.

[9] Min Zha et al. Data analysis and key science results of LHAASO-WCDA. 37th ICRC (2021), ID-1081.

[10] Yanjin Wang et al. The energy scale calibration using the Moon Shadow of LHAASO-WCDA detector. 37th ICRC (2021), ID-0897.

[11] Yuhua Yao et al. Search for very high energy gamma-ray emission from GRB190829A with LHASSO-WCDA1 triggerless data. 37th ICRC (2021), ID-0969.

[12] Zhiyong You et al. The energy spectrum of cosmic ray Proton and Helium above 100TeV measured by LHAASO experiment. 37th ICRC (2021), ID-1079.

[13] Ran Wang et al. Observing the gamma rays emission from the Markarian 421 with the LHAASO-WCDA. 37th ICRC (2021), ID-1103. 